\title{
The Media Use Analysis of New Generation Rural Migrant Workers`Ad- aptation to the Urban Society in China
}

\author{
Bin Li*1, a , Joyce Chen ${ }^{2, b}$ \\ ${ }^{1}$ Department of Communication, Jilin Agricultural University, Changchun 130118, Jilin Province, \\ China; \\ ${ }^{2}$ Department of Communication Studies, University of Northern lowa, Cedar Falls, IA50613,U.S. \\ aemail:10529129@qq.com, bemail:280339877@qq.com
}

Keywords: New generation rural migrant worker; culture adaptation; media effect; Uses and Gratification; difference

\begin{abstract}
Mass media has influenced the new generation rural migrant workers' culture adaptation based on their intimacy relationship of media use characteristics. This empirical study takes samples from Jilin province of Northeast China and analyzes this group's media use choice and differences through self-designed questionnaire scales. With the new generation migrant workers as audience subject, this study attempts to analyze mass media's influence on their adaptation to city life, demonstrate the needs gratification of job-hunting, daily life, interpersonal relationship, leisure and recreation. It also aims to identify the media use differences due to new generation rural migrant workers' diversity derived from demographic variables, contrast the use of traditional media with that of new media , and finally discuss the reasons for media use choice.
\end{abstract}

\section{Introduction and Literature review}

New generation migrant rural workers ${ }^{[1]}$ is defined as the generation in China who were born in the 1980's and 1990's and later moved from rural areas to live and work in the cities. As the definition indicates, this group of people has unique identity and cultural background. Since, many new generation migrant workers grew up in urban area following their family members, a large portion of them consider integrating into the urban society as their future life goal. According to State Statistics Bureau's report on supervising rural migrant workers, new generation is 125.28 million, accounting for $46.6 \%$ of the total group. Almost $1 / 3$ of the new generation group has high school education or above, which is $19.2 \%$ higher than their parent generation ${ }^{[2]}$.

Different from their parent generation who wish to return to the rural area after earning enough money, their need for acceptance and integration in accordance with the rules of modern urban cultural consciousness is greater than the need for identification with the rural society. "belong to the same culture is to say that they interpret the world in roughly the same ways and can express themselves, they thoughts and feelings about the world, in ways which will be understood by each oth-

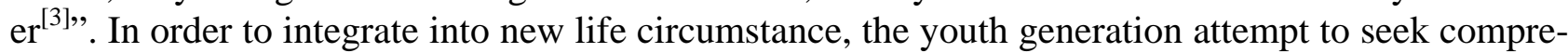
hensive ways to solve realistic questions. "On the gratifications individual seek, and on the roles the mass media play in meeting people`s needs, but it seems obvious that people do use media in varying ways [4]," Young rural migrant workers have become increasingly dependent on new media, which is to meet needs to obtain information and communicate with the outside world on a daily basis. According to statistics of CNNIC (2014), by December 2013, rural users have reached 0.177 billion, accounting for $28.6 \%$ of the total Internet users. The increasing scale of rural Internet users has made possible the enhanced new media literacy of young rural migrant workers ${ }^{[5]}$.

The core of this research literature discusses differences between the new generation and their parent generation. Age differences largely determine new generation migrant workers' advantaged media relationship, especially in using new digital media. Huang, Zhu, \& Wang ${ }^{[6.7]}$ pointed that mass media played important roles in information provision, speech rights and image formation which new generation migrant rural workers are in need of. Jiang ${ }^{[8]}$ suggested that young rural mi- 
grant workers' attempt to change the status quo may be dissimilated into psychological imbalance which may negatively influence social stability. Zhou ${ }^{[9]}$ used the stratified questionnaire to survey the Shanghai samples about the new media use and assessment of young rural migrant workers, finding that interpersonal communication, leisure and recreation have become the main functions of new generation migrant workers` media use. Mass media has facilitated the integration of new generation migrant rural workers into the urban life through new communication model ${ }^{[10]}$.

Many observers believe that the mass media generate a considerable amount of 'social teaching. Also, there is a lack of research analyzing the individual and social factors influencing young rural migrant workers' adaptation process. This empirical research is based on the theory of Uses-andGratifications. It takes the young rural migrant workers as audience subject and examines the influence of mass media on them during their cultural adaptation from rural to urban society, with due attention to rural migrant workers`needs. Katz, Blumler, and Gurevitch reviewed some early works on Uses and Gratification theory, Kartz pointed out that the focus of mass media research should shift to the audience. In view of this, we set up a research framework consisting of four dimensions, job-hunting, daily life, interpersonal communication, and leisure and recreation, to examine the needs of new generation migrant workers. By investigating the diversity of young rural migrant workers' choice of media use, we attempt to identify the differentiation that exists within the group. The concept of audience activity should be included in the study of Internet communication, and it has already been incorporated in one examination of the Cleveland Freenet ${ }^{[11]}$. In particular, through the interactive digital media, the young migrant workers try to seek opportunities to access the communication media and obtain the same life experiences as their peers in the cities. Their choice of using traditional media or new media depends on their needs as they face the strange urban society.

\section{Methods}

\subsection{Participants}

In this study, we have chosen the migrant workers' work locations, rural areas which they will be back on festival vacation, as well as train and bus stations to implement sample selection, in order to collect diverse sample statistics of new generation rural migrant workers. The test samples have been controlled in the age range of 16-30 years old, and the demographic variables are divided into gender, educational level, income level, registered residency, living residency, and profession classification to discriminate the media relationship discrepancy among new generation rural migrant workers. The study was implemented from January to March, 2015. Self-designed questionnaire was used to investigate 200 new generation rural migrant workers. Valid samples are 165(N=165). At the 95\% Confidence coefficient condition, maximum error has been no more than $\pm 3.1 \%$.

\subsection{Procedure}

\subsubsection{New generation rural migrant workers' media choice questionnaire}

A media choice self-compiled questionnaire was adopted, which includes 10 items, with 5 items about traditional media and 5 items about new media. The questionnaire used Likert 5-point scale assessment, from 'very unimportant' to 'very important'. The more score of the scale form, there is more need tendency. Confirmatory factor analysis: CFI=0.92, NFI=0.90 , TLI=0.97 , RMSEA $=0.06$, which indicates that the questionnaire has good statistics structure validity. The whole questionnaire coefficient of $\alpha$ is 0.88 , with traditional media and new media coefficients 0.85 and 0.81 , respectively.

\subsubsection{New generation rural migrant workers' media use needs questionnaire}

This media use need self-compiled questionnaire includes 23 items. Job-hunting involves 9 items, daily life involves 6 items, interpersonal relationship involves 4 items, and leisure and recreation involves 4 items. The questionnaire used Likert 5-point scale assessment, from 'very unimportant' to 'very important'. The more score of the scale form, the stronger the influence level. Confirmatory factor analysis: $\mathrm{CFI}=0.94$, NFI $=0.93$, TLI $=0.91$, RMSEA $=0.07$, which indicates 
that the questionnaire has good structural validity. The whole questionnaire coefficient of $\alpha$ is 0.89 , with traditional media and new media coefficients 0.85 and 0.81 , respectively.

\section{Results and Discussion}

\subsection{Differences in new generation rural migrant workers' media use choice}

By examining the new generation rural migrant workers through demographic variables, it is found that the interior of this group displays very different choices of media use.

\subsubsection{Media use choice of gender variable}

Independent-samples T test was adopted to investigate the media choice across different genders of new generation rural migrant workers. There is no significant difference in the choice of use between males and females regarding traditional media $(\mathrm{T}=-0.250, \mathrm{p}=0.083>0.05)$, but as to the choice of new media, males express significantly higher preferences than females $(\mathrm{t}=3.401$, $\mathrm{p}=0.001<0.01)$. The reason for the little difference of traditional media use is mainly due to the media terminal occupation level. New generation rural migrant workers have very low proportion of traditional media terminal use in their work places and daily contact. Another factor is that the cost of traditional terminals for both males and females is comparatively higher than that of the new media, which reduces the media availability for them.

\subsubsection{Media use choice on educational level variable}

F test was adopted to investigate the media use choice of new generation rural migrant workers with different educational levels.

Significant differences exist in terms of the educational level variable of new generation rural migrant workers' use choice of traditional media and new media $(\mathrm{F}=3.503, \mathrm{p}=0.032<0.05 ; \mathrm{F}=6.067, \mathrm{p}=0.003<0.01)$. The Posteriori comparison test was then conducted to account for the trend of traditional media use, and it was found that the below middle school group has higher degree of use than the above middle school group $\left(\mathrm{M}_{\mathrm{d} 1-}\right.$ ${ }_{2}=0.50, \mathrm{p}=0.012<0.05 ; \mathrm{M}_{\mathrm{d} 1-3}=0.51, \mathrm{p}=0.018<0.05$ ) , indicating that new generation migrant workers with low educational level are apt to choose traditional media than their high educational level counterparts. With respect to new media, along with the increase of educational level, the new media use choice tendency becomes higher $\left(\mathrm{M}_{\mathrm{d} 3-2}=0.32, \mathrm{p}<0.001 ; \mathrm{M}_{\mathrm{d} 3-1}=0.79, \mathrm{p}=0.041<0.05 ; \mathrm{M}_{\mathrm{d} 2-}\right.$ ${ }_{1}=0.47, \mathrm{p}=0.027<0.05$ ).

\subsubsection{Media use choice on monthly income variable}

$\mathrm{F}$ test was adopted to investigate the choice of media use among new generation rural migrant workers with different monthly income. The choice of traditional media and new media exhibits significant difference $(\mathrm{F}=4.228, \mathrm{p}=0.007<0.05 ; \mathrm{F}=8.538, \mathrm{p}=0.000<0.001)$ among new generation rural migrant workers with different income levels. The results of comparison test display that migrant workers whose income per month is under 1000 have higher level of traditional media use than the other three $\operatorname{groups}\left(\mathrm{M}_{\mathrm{d} 1-2}=0.66, \mathrm{p}=0.013<0.05 ; \mathrm{M}_{\mathrm{d} 1-3}=0.73, \mathrm{p}=0.005<0.01 ; \mathrm{M}_{\mathrm{d} 1-}\right.$ ${ }_{4}=0.90, \mathrm{p}=0.001<0.001$ ), and higher income level migrant workers` use choice of traditional media does not have statistically significant difference $\left(\mathrm{M}_{\mathrm{d} 2-3}=0.07, \mathrm{p}=0.661>0.05 ; \mathrm{M}_{\mathrm{d} 2-4}=0.24\right.$, $\left.\mathrm{p}=0.145>0.05 ; \mathrm{M}_{\mathrm{d} 3-4}=0.17, \mathrm{p}=0.26>0.05\right)$. As to the trend of new media, the group whose income per month is below $1000(\mathrm{RMB})$ is lower than the other three groups $\left(\mathrm{M}_{\mathrm{d} 1-2}=-0.89\right.$, $\left.\mathrm{p}=0.002<0.01 ; \mathrm{M}_{\mathrm{d} 1-3}=-0.88, \mathrm{p}=0.001<0.001 ; \mathrm{M}_{\mathrm{d} 1-4}=-1.31, \mathrm{p}=0.000<0.0010\right)$.

\subsubsection{Media use choice on registered residency variable}

F test was adopted to investigate the media choice of new generation rural migrant workers with different registered residency. Migrant workers with different registered residency have no significant diversity in the choice of traditional media and new media use $(F=1.774, p=0.137>0.05$; $\mathrm{F}=0.334, \mathrm{p}=0.855>0.05)$. 


\subsubsection{Media use choice on profession variable}

F test was adopted to investigate the choice of use among new generation rural migrant workers with different professions. It can be seen that there is no significant difference in the choice of using traditional media and new media among new generation rural migrant workers with different professions ( $\mathrm{F}=1.062, \mathrm{p}=0.383>0.05 ; \mathrm{F}=1.015, \mathrm{p}=0.411>0.05)$.

3.2 Regression prediction of new generation rural migrant workers' media use needs

\subsubsection{Prediction of media use needs of job-hunting}

Using demographic variable and media choice tendency (traditional media and new media) as independent variables and job-hunting as dependent variable, the Compel- Enter method was employed to make linear regression analysis. Demographic variable and media choice tendency can predict job-hunting dimension significantly $(\mathrm{F}=6.558, \mathrm{p}=0.000<0.001)$. Among the variables, gender, traditional media, and new media all predict the positive job-hunting dimension $(\beta 1=0.17$, $\mathrm{p}=0.02<0.05 ; \beta 2=0.45, \mathrm{p}=0.000<0.001 ; \beta 3=0.28, \mathrm{p}=0.001<0.05)$, and the traditional media $>$ new media $>$ gender.

During new generation rural migrant workers' adaptation to city life, the influence of media on their job-hunting is based on the demands of their culture and society in which they have to face the new circumstance. The conclusion of positive prediction is due to three factors. First, various forms of traditional media such as TV job-hunting program, recruitment information broadcast, and newspaper recruitment information bring about strong influence on the job-hunting of new generation migrant workers who trust media credibility. New media fails to become their important professional media choice largely because of its disadvantages in information accuracy and media supervision, which is a principal consideration in new generation migrant workers' media demand. Second, the policy guidance of the Chinese government in cultivating media public opinion is mainly focused on traditional media, which undertakes the media service function of job-hunting and rights protection, thus affecting new generation rural migrant workers' media use attitude and behavioral habit.

\subsubsection{Prediction of media use needs of daily life}

Using demographic variable and media choice tendency (traditional media and new media) as independent variables and daily life as dependent variable, the Compel- Enter method was adopted to make linear regression analysis. Demographic variable and media choice tendency can predict daily life dimension remarkably $(\mathrm{F}=5.158, \mathrm{p}=0.000<0.001)$. Among the variables, educational level, traditional media, and new media all predict the positive daily life dimension $(\beta 1=0.24$, $\mathrm{p}=0.002<0.01 ; \beta 2=0.16, \quad \mathrm{p}=0.035<0.05 ; \beta 3=0.35, \mathrm{p}=0.000<0.001$ ) , and new media $>$ educational level $>$ traditional media.

Analysis results indicate that new generation rural migrant workers with higher educational level attach more importance to media influence on daily life, and the influence of new media exceeds that of traditional media for them. There are two possible reasons. One of the reasons is that new generation rural migrant workers with higher educational level tend to have higher level of media literacy. They have broken through the passive, ritualized media use phase and learned to use media information effectively to bring benefits to their integration into urban life. The other reason is that new generation rural migrant workers with higher educational level have accumulated rich experience in consumption, life service, residency arrangement and presentation of themselves, which reduces the gap with urban peers.

\subsubsection{Prediction of media use needs of interpersonal relationship}

Using demographic variable and media choice tendency (traditional media and new media) as independent variables and interpersonal relationship as dependent variable, the Compel- Enter method was used to make linear regression analysis Demographic variable and media choice tendency can predict leisure and recreation dimension remarkably $(\mathrm{F}=3.298, \mathrm{p}=0.001<0.001)$. Among the variables only traditional media and new media can predict the positive leisure and recreation dimension $(\beta 1=0.29, \mathrm{p}=0.000<0.001 ; \beta 2=0.24, \mathrm{p}=0.005<0.01)$, and the new media $>$ traditional media. 
It is a significant component of urban mass culture to take advantage of mass media for recreation and leisure, which marks distinct differences from rural society of China whose leisure style mainly relies on interpersonal communication circumstances, so it is a representative difference between new generation rural migrant workers and the past migrant workers in information era. Beyond working time, new generation rural migrant workers have adopted mass media entertainment to substitute for playing cards, gambling, and acquaintance chats. Listening to music, watching video, playing online games, posting messages in online forums, reading e-books, chatting in the interest circle through virtual space, these new media uses are important factors in changing new generation rural migrant workers' leisure style. With the popularization of the network function of mobile phones with new generation rural migrant workers, the entertainment function of new media plays even more important roles for new generation rural migrant workers gradually, which can mediate changes in interpersonal relationship, life consumption, promotion of professional skills, and future planning. These changes occur based on new generation rural migrant workers' entertainment interest, and then perform more interactive and active roles in their leisure process.

\section{Conclusion}

To sum up, this survey study took the new generation rural migrant workers in Jilin province, China as quantitative test sample and investigated their media use. The results reflect the differences in media use tendency among new generation rural migrant workers. Different from most previous studies, this study adopted social layering study method and used layering standards such as gender, educational level, income level, registered residency, living residency, and profession to examine the media communication function with different groups of samples. It was found that mass media's influence on new generation rural migrant workers may not be integrative.

Through the regression analysis of the influence of mass media on new generation rural migrant workers, we can clearly distinguish the different effect mass media communication exerts through job-hunting, daily life, interpersonal relationship, leisure and recreation dimension based on the use demand of this group in adapting to the urban unfamiliar circumstance. Although new generation rural migrant workers are highly dependent on new media, they have cultivated their media use choice and habits according to their demands, not just decided by the new media preference. Media communication gratification for these migrant workers is a process, which develops gradually according to the changes in their urban living status. It is a reflection of their demands in the transition from rural to urban culture.

\section{Acknowledgement}

In this paper, the research was sponsored by Social Science Project of Jilin Provincial Education Department (Project No. jiaokewen105) and Vocational Education Project of Jilin Provincial Education Department (Project No. 2015cyz328).

\section{References}

[1]China State Council. The advice on the promotion urban-rural development and cultivating the basement of agricultural development of China State Council[R]. Beijing: People Press.2010.

[2]National Bureau of China. National migrant workers monitor report in 2013[EB/OL]. http://www.gov.cn/xinwen/2014/05/12/content_2677897.htm.

[3]Hall, Stuart. Representation: Cultural representations and signifying practices[M]. London: Sage.1997.2-3.

[4]Berger Asa Arthur. Sociological analysis Media analysis techniques[M]. Thousand Oaks, CA: Sage. 2012. 115-116. 
[5]China Internet Network Information Center. The 33th China internet development statistics report in 2014[R]. 2014.

[6]Yunhui Zhu. \& Yanlong Wang. Construction of media touch and public service platform of new generation migrant rural workers [J]. Press Circles. 2011(5):62-63.

[7]Junhua Huang. Media strategy of new generation migrant socialization. Press Circles. 2011 (4):62-63.

[8]Shenghong Jiang.The problem and strategy research of China new generation migrant rural workers[J]. Lanzhou academic Journal. 2011(3):89-92.

[9]Baohua Zhou. The empirical research of new media using and assessment of new generation in Shanghai[J]. Journalism Quarterly, 2011(2):.145-150.

[10]Wanlin Song. Promotion model research between the mass media on new generation migrant rural workers[J].News Knowledge. 2011(12): 7-9.

[11]Swift, C.R. Audience activity in computer-mediated communication, Unpublished doctoral dissertation[M]. Indiana University, Bloomington, IL. 1989. 